\title{
RISCO NUTRICIONAL EM CIRURGIA AVALIADO PELO ÍNDICE DE MASSA CORPORAL AJUSTADO OU NÃO PARA PACIENTES IDOSOS
}

\author{
Alberto BICUDO-SALOMÃO, José Eduardo de AGUILAR-NASCIMENTO e \\ Cervantes CAPOROSSI
}

RESUMO - Racional - O índice de massa corporal vem sendo usado indistintamente entre idosos ou não para a avaliação do estado nutricional. A hipótese deste trabalho é a de que em pacientes idosos um ponto de corte mais alto para o índice de massa corporal pode ter maior validade na aferição do risco nutricional em cirurgia. Objetivo - Avaliar a morbimortalidade em pacientes cirúrgicos idosos comparada a pacientes mais jovens e sua associação com valores baixos de índice de massa corporal utilizando-se um ponto de corte convencional $\left(18,5 \mathrm{~kg} / \mathrm{m}^{2}\right)$ e outro adaptado para pacientes com idade avançada $\left(24 \mathrm{~kg} / \mathrm{m}^{2}\right)$. Pacientes e métodos - Um mil e novecentos e doze pacientes foram divididos em dois grupos: $<65$ anos $(\mathrm{n}=1627)$ e $\geq 65$ anos $(\mathrm{n}=285)$. Comparou-se nos dois grupos o tempo de internação e a morbimortalidade. Pacientes com idade $\geq 65$ anos foram estratificados segundo o índice de massa corporal $<18,5 \mathrm{~kg} / \mathrm{m}^{2}$ ou $<24 \mathrm{~kg} / \mathrm{m}^{2}$, e analisados quanto a complicações pós-operatórias, reoperações, óbito e tempo de internação. Resultados - O tempo de internação foi maior em pacientes com idade $\geq 65$ anos (6 [1-75] dias vs. 4 [1-137] dias), assim como as complicações pós-operatórias (37/285; 13,0\% vs. 109/1627; 6,7\%; OR 2,1; IC95\% 1,40-3,09) e óbitos (15/285; 5,3\% vs. 34/1627; 2,1\%; OR 2,6; IC95\% 1,40-4,84). Em pacientes com índice de massa corporal $\leq 24 \mathrm{~kg} / \mathrm{m}^{2}$, o tempo de internação (8 [1-75] dias vs. 4 [1-43] dias), complicações pós-operatórias (28/152; 18,4\% vs. 9/133; 6,8\%; OR 3,1; IC95\% 1,41-6,86) e reoperações $(16 / 152$; $10,5 \%$ vs. 3/133; $2,2 \%$; OR 5,1; IC95\% 1,45-17,91) mostraram-se maiores. Utilizando-se como ponto de corte o índice de massa corporal de $18,5 \mathrm{~kg} / \mathrm{m}^{2}$, não houve diferenças. Conclusões - A morbimortalidade é alta em pacientes cirúrgicos de idade avançada. Nestes, o índice de massa corporal abaixo de $24 \mathrm{~kg} / \mathrm{m}^{2}$ mostrou melhor associação com a ocorrência de complicações pós-operatórias, reoperações e tempo prolongado de internação.

DESCRITORES - Estado nutricional. Desnutrição. Nutrição do idoso. Procedimentos cirúrgicos operatórios. Índice de massa corporal. Tempo de internação. Idoso.

\section{INTRODUÇÃO}

A desnutrição é um problema de significativa importância em pacientes cirúrgicos. Relaciona-se a maiores custos hospitalares, maior tempo de internação, maior incidência de complicações e óbito pós-operatório( ${ }^{(1)}$. O idoso é particularmente mais susceptível a déficits nutricionais. A má nutrição que ocorre nesses pacientes é de causa multifatorial: deve-se a alterações fisiológicas do envelhecimento, às condições socioeconômicas, comorbidades e à interação entre nutrientes e medicamentos. Isso torna a avaliação nutricional no paciente idoso peculiar, devendo ser feita de maneira padronizada e criteriosa $^{(15)}$. Deve-se ainda ressaltar que a idade afeta muitos dos métodos comumente usados de análise do status nutricional ${ }^{(13)}$. Tais fatores fazem com que seja difícil definir, com precisão, desnutrição nesses pacientes ${ }^{(12)}$.
O índice de massa corporal (IMC), determinado pela relação entre o peso corporal em kg e estatura em $\mathrm{m}^{2}$ é, na prática diária, amplamente utilizado. Além de ser de fácil execução é, especialmente em estudos populacionais, bom indicador do estado nutricional por sua boa correlação com a massa corporal $(\mathrm{r} \approx 0,80)$ e baixa correlação com a estatura ${ }^{(8)}$. São considerados valores normais para o IMC entre $18,5 \mathrm{~kg} / \mathrm{m}^{2}$ e $25 \mathrm{~kg} / \mathrm{m}^{2}$. Valores acima ou abaixo destes limites são indicativos de risco nutricional ${ }^{(11)}$. Todavia, nos idosos, esse índice classicamente têm sido considerado indicador pobre para a avaliação de riscos ${ }^{(14)}$. O emprego do IMC nesse grupo apresenta dificuldades por não levar em consideração as alterações da composição e estrutura do corpo que lhes são típicas, como decréscimo da estatura, acúmulo de tecido adiposo, redução de massa corporal magra e da quantidade de água no organismo ${ }^{(3,9)}$.

Departamento de Clínica Cirúrgica, Universidade Federal do Mato Grosso, Cuiabá, MT. 
Por esses motivos, nas últimas décadas surgiram estudos visando estabelecer pontos de corte mais altos para o IMC em idosos e sua correlação com outros métodos de avaliação nutricional( $^{(8)}$. BRAY ${ }^{(4)}$ classifica como desejável em pacientes acima de 65 anos o IMC entre $24-29 \mathrm{~kg} / \mathrm{m}^{2}$. LIPSCHITZ ${ }^{(13)}$ reforça que nesses pacientes, valores de IMC inferiores a $22 \mathrm{~kg} / \mathrm{m}^{2}$ são indicativos de significante déficit de nutricional. RANHOFF et al. ${ }^{(16)}$, recomendam que o IMC abaixo de $23 \mathrm{~kg} / \mathrm{m}^{2}$ em idosos com doenças agudas, têm alta sensibilidade na detecção de desnutrição ou risco de desnutrição, devendo ser seguido por outras ferramentas de rastreamento, visando a pronta identificação e correção de déficits. Observa-se portanto, que não há consenso do que seja IMC elevado ou baixo para o indivíduo idoso ${ }^{(19)}$. A Organização Mundial da Saúde sugere para a avaliação do idoso a utilização dos pontos de corte propostos para o adulto jovem (IMC $<18,5 \mathrm{~kg} / \mathrm{m}^{2}=$ magreza; IMC $\geq 30 \mathrm{~kg} / \mathrm{m}^{2}=$ obesidade), muito embora as evidências atuais estejam mostrando que o emprego de valores superiores sejam mais adequados ${ }^{(10)}$.

Há poucos estudos na literatura mostrando a relação entre valores de IMC em idosos e morbimortalidade em pacientes cirúrgicos. Para esses doentes são escassos, em nosso meio, dados de medida de freqüência em populações hospitalares e avaliações de riscos aos quais os mesmos estão submetidos. A partir disso, o objetivo desse estudo foi avaliar em idosos internados em enfermaria de cirurgia geral de um hospital universitário na região centro-oeste do Brasil, a incidência de complicações cirúrgicas, mortalidade e o tempo de internação, comparando-se com pacientes mais jovens. Secundariamente, objetivou-se correlacionar tais variáveis com valores baixos de IMC, utilizando-se como ponto de corte, dois valores distintos: um convencional e outro adaptado para pacientes com idade avançada.

\section{PACIENTES E MÉTODO}

Foram estudados prospectivamente 1912 pacientes internados na enfermaria do Departamento de Clínica Cirúrgica do Hospital Universitário Júlio Muller, Universidade Federal do Mato Grosso, Cuiabá, MT, dentre os anos de 2002 a 2004.

Foram divididos em dois grupos conforme a idade: menor de 65 anos $(n=1627)$ e maior ou igual a 65 anos $(n=285)$. Estes últimos doentes foram divididos nos grupos etários de 65 a 75 anos $(n=226)$ e maiores de 75 anos $(n=59)$. Foram comparados o tempo de internação, complicações pós-operatórias e óbitos. Os dados demográficos dos pacientes estudados são vistos na Tabela 1.
Os pacientes com idade maior ou igual a 65 anos foram estratificados segundo o IMC $\leq 18,5 \mathrm{~kg} / \mathrm{m}^{2}$ ou $>18,5 \mathrm{~kg} / \mathrm{m}^{2}$ (ponto de corte de $18,5 \mathrm{~kg} / \mathrm{m}^{2}$ ) e $\leq 24 \mathrm{~kg} / \mathrm{m}^{2}$ ou $>24 \mathrm{~kg} / \mathrm{m}^{2}$ (ponto de corte de $24 \mathrm{~kg} / \mathrm{m}^{2}$ ) (Tabela 2). As variáveis de resultado estudadas foram complicações pós-operatórias (infecção de sítio cirúrgico, sangramentos pós-operatório, deiscências e fístulas), reoperações, óbito e tempo de internação.

TABELA 2 - Pacientes com idade maior ou igual a 65 anos, segundo ponto de corte do índice de massa corporal $\left(\mathrm{kg} / \mathrm{m}^{2}\right)$

\begin{tabular}{lccccc}
\hline & \multicolumn{4}{c}{ Ponto de corte de IMC $\left(\mathrm{kg} / \mathrm{m}^{2}\right)$} \\
\cline { 2 - 3 } \cline { 5 - 6 } & \multicolumn{2}{c}{$\mathbf{1 8 , 5}$} & \multicolumn{2}{c}{24} \\
\cline { 2 - 3 } & $\mathbf{1 8 , 5}$ & $>18,5$ & & $\leq 24$ & $>24$ \\
Pacientes (n, \%) & $29(10,2 \%)$ & $256(89,8 \%)$ & & $152(53,3 \%)$ & $133(46,7 \%)$ \\
Idade (anos) & $78,7( \pm 9,55)$ & $71,6( \pm 5,22)$ & & $73,5( \pm 7,86)$ & $70,9( \pm 4,86)$ \\
Neoplasia maligna (n, \%) & $16(55,1 \%)$ & $82(32 \%)$ & & $65(42,7 \%)$ & $33(24,8 \%)$ \\
\hline
\end{tabular}

Para a análise dos dados obtidos, foi utilizado o pacote de programas estatísticos Epi-Info, versão 2002. A variável tempo de internação foi avaliada quanto à distribuição normal com o teste de Kolmogorov-Smirnov e homogeneidade de variâncias pelo teste de Levene, sendo expressa na forma de mediana e variação. Para comparação entre os grupos estudados foi utilizado o teste nãoparamétrico de Mann-Whitney. As variáveis qualitativas (ocorrência de complicações pós-operatórias, reoperações e óbitos) foram analisadas entre os diferentes grupos utilizando-se o teste qui-quadrado ou o teste exato de Fisher, conforme o tamanho da amostra estudada. Foi adotado como índice de significância estatística o valor de $P<0,05$. Como medida da força de associação, foi realizado o cálculo do odds-ratio (OR), com intervalo de confiança de 95\% (IC95\%).

\section{RESULTADOS}

Durante os anos de 2002 a 2004, 285 pacientes com idade maior ou igual a 65 anos foram internados na enfermaria de clínica cirúrgica do Hospital Universitário Júlio Muller, correspondendo a $14,9 \%$ do total de internados nesse período $(n=1912)$.

O tempo de internação foi significantemente maior no grupo de pacientes com idade maior ou igual a 65 anos (6 [1-75] dias vs. 4 [1-137] dias, $P<0,001)$. Estes apresentaram ainda percentual de complicações pós-operatórias $(37 / 285 ; 13,0 \%$ vs. $109 / 1627 ; 6,7 \%$; OR 2,1 ; IC95\% 1,40-3,09) e percentual de óbitos $(15 / 285 ; 5,3 \%$ vs. $34 / 1627 ; 2,1 \%$; OR 2,6; IC95\% 1,40 $4,84)$ significantemente maior $(P<0,05)$ que os pacientes com idade inferior a 65 anos. O Gráfico 1 ilustra a razão de chances

TABELA 1 - Dados demográficos de 1912 pacientes, segundo a faixa etária

\begin{tabular}{|c|c|c|c|c|c|c|c|c|}
\hline \multirow[b]{2}{*}{ Sexo } & \multicolumn{4}{|c|}{$\begin{array}{c}\text { Idade }<65 \text { anos } \\
(\mathrm{n}=1627)\end{array}$} & \multicolumn{4}{|c|}{$\begin{array}{c}\text { Idade } \geq 65 \text { anos } \\
(\mathrm{n}=285)\end{array}$} \\
\hline & \multicolumn{2}{|c|}{ Masculino } & \multicolumn{2}{|c|}{ Feminino } & \multicolumn{2}{|c|}{ Masculino } & \multicolumn{2}{|c|}{ Feminino } \\
\hline & \multicolumn{2}{|c|}{$762(46,8 \%)$} & \multicolumn{2}{|c|}{$865(53,2 \%)$} & \multicolumn{2}{|c|}{$107(37,5 \%)$} & \multicolumn{2}{|c|}{$178(62,5 \%)$} \\
\hline \multirow[t]{2}{*}{ Raça } & Branco & Negro & Pardo & Amarelo & Branco & Negro & Pardo & Amarelo \\
\hline & $717(44,06 \%)$ & $296(18,19 \%)$ & $592(36,08 \%)$ & $22(1,35 \%)$ & $129(45,4 \%)$ & $56(19,7 \%)$ & $96(33,8 \%)$ & $4(1,4 \%)$ \\
\hline Idade (anos) & \multicolumn{4}{|c|}{$40,92( \pm 13,52)$} & \multicolumn{4}{|c|}{$72,30( \pm 13,54)$} \\
\hline $\begin{array}{l}\text { Neoplasia } \\
\text { maligna }\end{array}$ & \multicolumn{4}{|c|}{$201(12,35 \%)$} & \multicolumn{4}{|c|}{$98(34,38 \%)^{*}$} \\
\hline
\end{tabular}

* $P<0,05$ vs. idade $<65$ anos 
(OR) e intervalo de confiança de 95\% (IC95\%) dos pacientes com idade $\geq 65$ anos apresentarem complicações ou óbito no pós-operatório - o ponto em destaque $(\bullet)$ mostra o valor de OR para cada variável com as linhas horizontais correspondendo

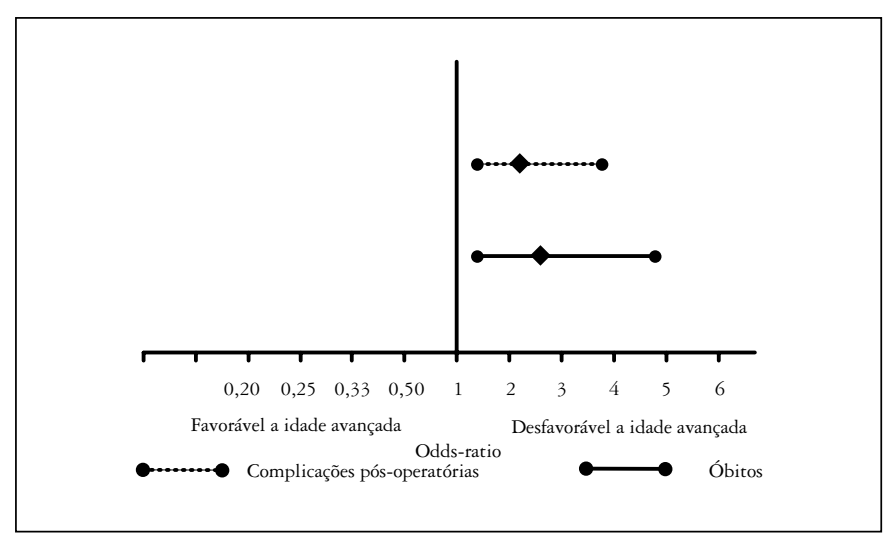

GRÁFICO 1 - Odds-ratio e intervalo de confiança de 95\% (IC95\%) para complicações e óbitos pós-operatórios associados a idade avançada ( $\geq 65$ anos) no eixo das abscissas aos respectivos intervalos de confiança de $95 \%$; tais valores acima de 1 , conforme destacado no gráfico, determinam que a idade $\geq 65$ anos representou fator de risco (desfavorável) para morbimortalidade operatória. Calculandose ainda o produto de riscos cruzados entre os grupos etários, estratificado pela ocorrência de neoplasia maligna, observou-se OR relacionado ao risco de complicações pós-operatórias de 3,0 (IC95\% 2,40-3,80) e de óbito 3,5 (IC95\% 2,70-4,53).

Analisando-se apenas pacientes idosos, quando se comparou o grupo etário de 65 a 75 anos com pacientes com idade superior a 75 anos, não houve diferenças estatísticas em relação a tempo de internação $(10,42 \pm 10,64$ vs. $8,64 \pm 10,79, P=0,257)$, complicações pós-operatórias $(32 / 226 ; 14,2 \%$ vs. $04 / 59$; $6,8 \%, P=0,19)$ e mortalidade $(13 / 226 ; 5,8 \%$ vs. $02 / 59 ; 3,4 \%$, $P=0,37)$. Em relação ao índice de massa corporal, $29 / 285$ $(10,2 \%)$ foram classificados como de baixo IMC quando adotado ponto de corte de $18,5 \mathrm{~kg} / \mathrm{m}^{2}$. Todavia, com ponto de corte de $24 \mathrm{~kg} / \mathrm{m}^{2}$, o número de pacientes classificados como tendo baixo IMC $(152 / 285 ; 53,3 \%)$ foi significantemente maior $(P<0,05)$.

Com ponto de corte de $24 \mathrm{~kg} / \mathrm{m}^{2}$, o tempo de internação foi significantemente maior nos paciente com baixo IMC (8 [1-75]

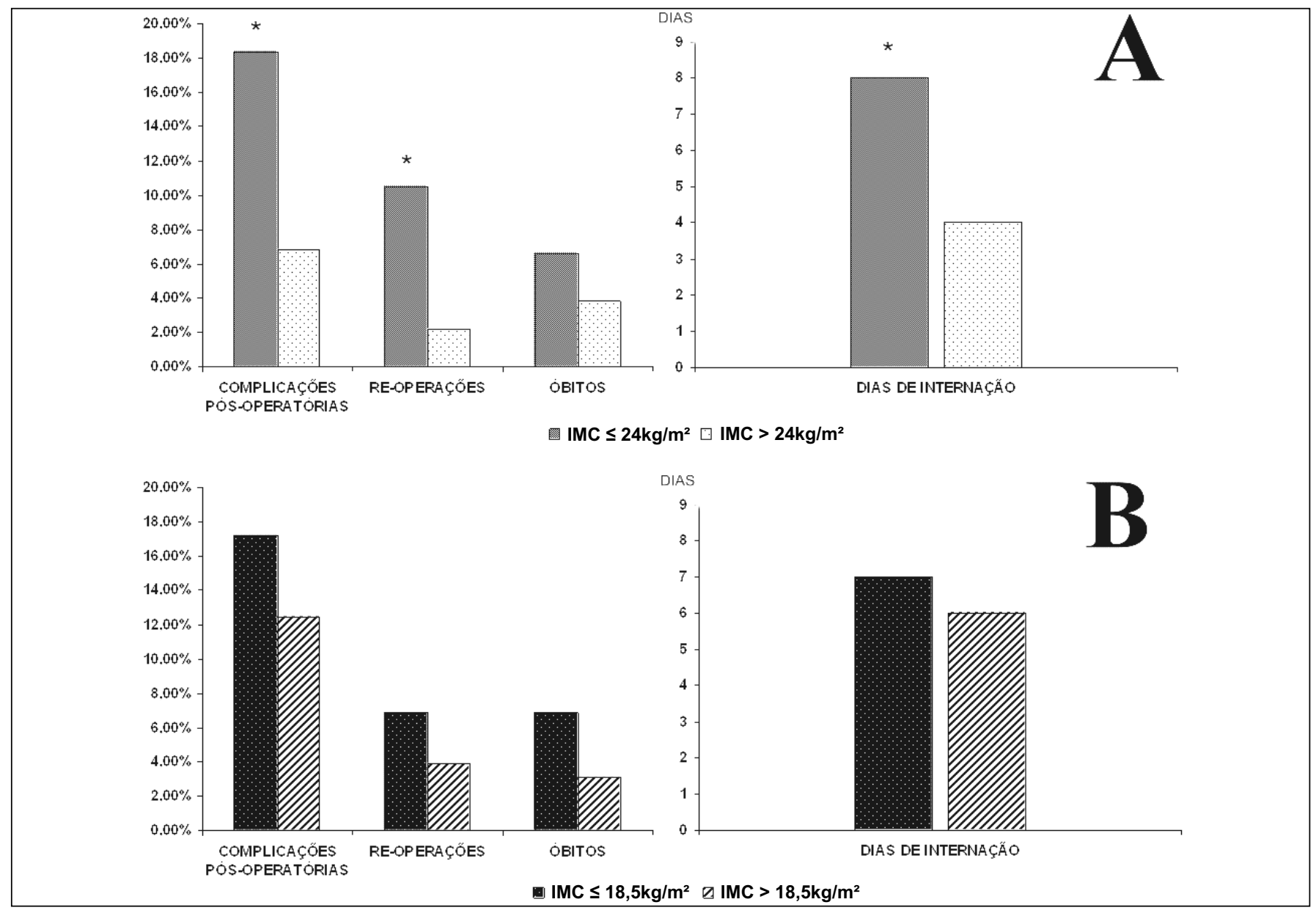

${ }^{*} P<0,05$ vs. grupo IMC $>24 \mathrm{~kg} / \mathrm{m}^{2}$

GRÁFICO 2 - Tempo de internação, morbidade e mortalidade operatória de pacientes idosos (idade $\geq 65$ anos) em função do baixo IMC estratificado pelo ponto de corte de $24 \mathrm{~kg} / \mathrm{m}^{2}$ (A) ou $18 \mathrm{~kg} / \mathrm{m}^{2}$ (B) 
dias vs. 4 [1-43] dias, $P<0,05)$. Já usando-se ponto de corte de $18,5 \mathrm{~kg} / \mathrm{m}^{2}$, não houve diferença estatística (7 [1-41] dias vs. 6 [1-75] dias, $P=0,28$ )

Com ponto de corte de $24 \mathrm{~kg} / \mathrm{m}^{2}$, o percentual de complicações pós-operatórias $(28 / 152 ; 18,4 \%$ vs. $9 / 133 ; 6,8 \%$; OR 3,$1 ;$ IC95\% $1,41-6,86)$ e reoperações $(16 / 152 ; 10,5 \%$ vs. $3 / 133 ; 2,2 \%$; OR 5,1 ; IC95\% 1,45-17,91) foi significantemente maior $(P<0,05) \mathrm{em}$ pacientes de baixo IMC. Com ponto de corte de $18,5 \mathrm{~kg} / \mathrm{m}^{2}$, não houve diferença em relação a complicações pós-operatórias $(5 / 29$; $17,2 \%$ vs. $32 / 256 ; 12,5 \% ; P=0,55)$ e percentual de reoperações $(2 / 29 ; 6,9 \%$ vs. $10 / 256 ; 3,9 \% ; P=0,35)$. O percentual de óbitos não mostrou diferença estatística relacionada ao baixo IMC seja estratificado por $24 \mathrm{~kg} / \mathrm{m}^{2}(10 / 152 ; 6,6 \%$ vs. $5 / 133 ; 3,8 \%$; $P=0,42)$, ou estratificado pelo IMC de $18,5 \mathrm{~kg} / \mathrm{m}^{2}(2 / 29 ; 6,9 \%$ vs. $8 / 256 ; 3,1 \% ; P=0,27$ ) (Gráfico 2 ).

\section{DISCUSSÃO}

Ao longo das últimas décadas, a população de indivíduos idosos vem apresentando crescimento progressivo. No ano $2000,12,4 \%$ da população dos EUA (cerca de 35000000 de pessoas) era formada por indivíduos com idade superior a 65 anos $^{(10)}$. Conseqüentemente, torna-se nítido o aumento do seu número em internações hospitalares ${ }^{(21)}$, acarretando aumento no número de operações. De 1980 até 1996, o percentual de operações em pacientes com idade acima de 65 anos aumentou de $19 \%$ para $36 \%{ }^{(18)}$.

No presente estudo, cerca de $15 \%$ das internações cirúrgicas foram de pacientes com idade maior ou igual a 65 anos. Os idosos apresentaram maior tempo de permanência hospitalar em relação a pacientes mais jovens, implicando em maiores custos hospitalares e maior morbimortalidade. Realmente observou-se risco duas vezes maior de complicações pós-operatórias e 2,6 vezes maior de óbitos em pacientes com idade avançada, quando comparados aos mais jovens. Tais achados estão de acordo com outros trabalhos da literatura, mostrando que, muito embora a morbidade e a mortalidade operatória em pacientes idosos venham diminuindo com o tempo ${ }^{(20)}$ a idade avançada tem correlação positiva com complicações ${ }^{(18)}$.

Deve-se considerar nesses resultados o aumento de co-morbidades e doenças consumptivas, em especial neoplasias que acompanham os pacientes idosos, observando-se correlatamente percentual significativamente maior de neoplasias malignas. Analisando-se a ocorrência de complicações pós-operatórias e óbitos, estratificada pela presença de neoplasia maligna nos dois grupos etários, observou-se aumento para 3 e 3,5 vezes desses riscos, respectivamente.

Outro importante aspecto diz respeito à sarcopenia no idoso. Este termo, inicialmente proposto por ROSENBERG ${ }^{(17)}$, refere-se à redução da massa muscular esquelética, com perda de sua força e função, relacionada à idade avançada ${ }^{(22)}$. Achados recentes sugerem que a sarcopenia seria um processo multifatorial onde estão envolvidos a inatividade física, remodelamento de unidades motoras, decréscimo de níveis hormonais e da síntese protéica $^{(5)}$. Aparentemente, inicia-se na quarta década de vida e tende a se acelerar após a idade de aproximadamente $75 \operatorname{anos}^{(23)}$.
Na presente casuística, pacientes com idade de 65 a 75 anos não tiveram diferenças estatísticas relacionadas ao tempo de internação, complicações pós-operatórias e óbitos em relação a pacientes com idade superior a 75 anos. Todavia, os resultados observados comparando-se doentes com idade $\geq 65$ anos aos mais jovens fazem acreditar que a sarcopenia também influencie nos resultados operatórios em idosos, o que se relaciona ao impacto da idade na performance fisiológica individual desses pacientes. Novos estudos serão necessários para explorar os reais efeitos da sarcopenia em pacientes cirúrgicos com idade avançada.

$\mathrm{O}$ estado nutricional mostrou ser fator relevante na morbidade operatória. A ocorrência de baixo IMC, tendo como ponto de corte o valor de $24 \mathrm{~kg} / \mathrm{m}^{2}$ esteve relacionada com maior tempo de internação, maior risco de complicações pós-operatórias e de reoperações em pacientes com idade avançada. A precisão de tais resultados foi afetada pelo intervalo de confiança relativamente grande observado, o que demonstra a necessidade de investigações epidemiológicas que englobem populações maiores. O mesmo pode ser aplicado à variável óbito que, neste estudo, não mostrou relação com o baixo IMC.

Não obstante, os achados desta série mostram-se de acordo com a tendência atual de se adotar pontos de corte de IMC mais altos em idosos ${ }^{(4,13,19)}$. O percentual de baixo IMC obtido com o ponto de corte convencional esteve muito abaixo do que se espera, em termos de prevalência nacional de desnutrição em pacientes cirúrgicos. Já os valores vistos com ponto de corte de $24 \mathrm{~kg} / \mathrm{m}^{2}$ mostraram-se muito mais compatíveis com a realidade ${ }^{6}$, ${ }^{24)}$. Os resultados deste estudo mostram que o ponto de corte para desnutrição proposto para adultos jovens (IMC $<18,5 \mathrm{~kg} / \mathrm{m}^{2}$ ), quando aplicado a pacientes idosos, não mostrou relação com nenhuma das variáveis estudadas. BECK e OVENSEN ${ }^{(2)}$, após extensa revisão de literatura sobre fatores de risco nutricional e IMC em idosos internados, propõem a elevação do ponto de corte para baixo IMC nesses pacientes de $18,5-20 \mathrm{~kg} / \mathrm{m}^{2}$ (conforme preconizado por muitos protocolos) para $24 \mathrm{~kg} / \mathrm{m}^{2}$, o que teria maior relação com complicações decorrentes da desnutrição. Em pacientes cirúrgicos, o IMC talvez não seja a melhor ferramenta na avaliação do estado nutricional ${ }^{(7)}$. No entanto, em especial no paciente idoso, o IMC pode servir como fator de alerta, apontando para o uso de outras formas mais efetivas de avaliação do estado nutricional, identificando com maior eficácia populações sob risco, afim de que medidas de suporte sejam tomadas ${ }^{(19)}$.

Conclui-se, portanto, que a incidência de complicações pósoperatórias, mortalidade e o tempo de internação são altos em pacientes cirúrgicos de idade avançada, quando comparados com pacientes mais jovens. Na população estudada, o ponto de corte do índice de massa corporal de $24 \mathrm{~kg} / \mathrm{m}^{2}$ mostrou maior relação com a ocorrência de complicações pós-operatórias e tempo prolongado de permanência hospitalar em pacientes com idade maior ou igual a 65 anos. Isto demonstra a necessidade de uma avaliação diferenciada nos idosos para a indicação de suporte nutricional precoce quando necessário, visando minimizar os riscos inerentes a essa faixa etária. 
Bicudo-Salomão A, Aguilar-Nascimento JE, Caporossi C. Nutritional risk in surgery evaluated by body mass index adjusted or not to elderly patients. Arq Gastroenterol. 2006;43(3):219-23.

ABSTRACT - Background - Standard body mass index cut-off points for malnutrition are routinely used for adults independently of their age. The hypothesis of this study was that a cut-off point higher than the usual for the diagnosis of malnutrition might be more precise to access the nutritional risk of aged surgical patients. Aim - To evaluate the morbimortality in aged surgical patients and its association with nutritional status assessed by body mass index using either the standard and a higher cut-off point for malnutrition. Patients and methods - All patients admitted for operative procedures $(\mathrm{n}=1,912)$ were allocated to either two groups: $<65$ years $(\mathrm{n}=1,627)$ or $\geq 65$ years old $(\mathrm{n}=285)$. The body mass index was used to access the nutritional status. Two different cut-off points for malnutrition $\left(18.5 \mathrm{or} 24 \mathrm{~kg} / \mathrm{m}^{2}\right)$ were used in the group of older patients. Endpoints of the study were length of stay, morbidity and mortality. Results - Length of stay was higher in patients over 65 years (6 [1-75] days vs. 4 [1-137] days). Both postoperative complications (37/285; 13.0\% vs. 109/1627; 6,7\%; OR 2,1; IC95\% 1,40-3,09) and deaths $(15 / 285 ; 5.3 \%$ vs. 34/1627; 2,1\%; OR 2,6; IC95\% 1,40-4,84) were most common in the older group. Using a higher cut-off $\left(24 \mathrm{~kg} / \mathrm{m}^{2}\right)$, the length of stay ( 8 [1-75] days vs. 4 [1-43] days), postoperative complications $(28 / 152 ; 18.4 \%$ versus 9/133; 6.8\%; OR 3,1; IC95\% 1.41-6.86) and re-operations (16/152; $10.5 \%$ versus $3 / 133 ; 2.2 \%$; OR 5.1; IC95\% $1.45-17.91)$ were greater in malnourished patients. However these correlations were not statistically significant with the cut-off point for malnutrition in $18.5 \mathrm{~kg} / \mathrm{m}^{2}$. Conclusions - Morbimortality is higher in surgical patients over 65 years old. In these, the cut-off point set in $24 \mathrm{~kg} / \mathrm{m}^{2}$ was most associated with the occurrence of postoperative complications, re-operations and length of stay.

HEADINGS - Nutritional status. Malnutrition. Elderly nutrition. Surgical procedures, operative. Body mass index. Lenght of stay. Aged.

\section{REFERÊNCIAS BIBLIOGRÁFICAS}

1. Aguilar-Nascimento JE, Caporossi C, Serra MC. Implicações da desnutrição em cirurgia. Rev Col Bras Cir. 1991;28:193-7.

2. Beck AM, Ovesen L. At which body mass index and degree of weight loss should hospitalized elderly patients be considered at nutritional risk? Clin Nutr. 1998;17:195-8

3. Bedogni G, Pietrobelli A, Heymsfield SB, Borghi A, Manzieri AM, Morini P. Is body mass index a measure of adiposity in elderly women? Obes Res. 2001;9:17-20

4. Bray GA. Classificação e avaliação das obesidades. Rio de Janeiro: Interamericana; 1989.

5. Bross R, Javanbakht M, Bhasin S. Anabolic intervations for aging-associated sarcopenia J Clin Endocrinol Metab. 1999;84:3420-30.

6. Correia MI, Waitzberg DL. The impact of malnutrition on morbidity, mortality, length of hospital stay and costs evaluated through a multivariate model analysis. Clin Nut. 2003;22:235-9.

7. Dock DB, Aguilar-Nascimento JE, Balsser M. Índice de massa corporal e peso teórico subestimam diagnóstico nutricional em pacientes cirúrgicos. Rev Bras Nutr Clin. 2005 [no prelo].

8. Dos Santos DM, Sichieri R. Índice de massa corporal e indicadores antropométricos de adiposidade em idosos. Rev Saúde Pública. 2005;39:163-8.

9. Gallagher D, Visser M, Sepúlveda D, Pierson RN, Harris T, Heymsfield SB. How useful is body mass index for comparason of body fatness across age, sex, and ethnic groups? Am J Epidemiol. 1996;146:228-39.

10. Hetzel L, Smith A. The 65 years and over population: 2000. Washington, DC: Census 2000 brief, C2KBR/01-10, US Census Bureau; 2001.

11. James WPT, Ferro-Luzzi A, Waterlow JC. Definition of chronic energy deficiency in adults. Eur J Clin Nutr. 1988;42:969-81.

12. Kuzuya M, Kanda S, Koike T, Suzuki Y, Iguchi A. Lack of correlation between total lymphocyte count and nutritional status in the elderly. Clin Nutr. 2005;24:427-32. 13. Lipschitz DA. Screening for nutritional status in the elderly. Prim Care. 1994;21:57-67.

14. Lohman TG, Roche AF, Martorell R, editors. Anthropometric standardization reference manual. Champaign, Il: Human Kinetics Books; c1988.

15. Marchini JS, Ferriolli E, Moriguti JC. Suporte nutricional no paciente idoso: definição, diagnóstico, avaliação e intervenção. Medicina (Ribeirão Preto). 1998:31:54-61.

16. Ranhoff AH, Gjoen AU, Mowe M. Screening for malnutrition in elderly acute medical patients: the usefulness of MNA-SF. J Nutr Health Aging. 2005;9:221-5.

17. Rosenberg IH. Sarcopenia: origins and clinical relevance. J Nutr. 1997;127: 990S-1S.

18. Rosenthal RA, Zenilman ME. Cirurgia no idoso. In: Sabiston, tratado de cirurgia: as bases biológicas da prática cirúrgica moderna. Beauchamps, Evers \& Mattox, $16^{\mathrm{a}}$ Edição, Rio de Janeiro: Guanabara Koogan, 2002.p 243-66.

19. Sampaio LR. Avaliação nutricional e envelhecimento. Rev Nutr. 2004;17:507-14.

20. Thomas DR, Ritchie CS. Preoperative assessment of older adults. J Am Geriatr Soc. 1995:43:811-21.

21. Tierney AJ. Undernutrition and elderly hospital patients: a review. J Adv Nurs. 1996;23:228-36.

22. Vandervoort AA, Symons TB. Functional and metabolic consequences of sarcopenia. Can J Appl Physiol. 2001;26:90-101.

23. Waters DL, Baumgartner RN, Garry PJ. Sarcopenia: current perspectives. J Nutr Health Aging. 2000;4:133-9.

24. Waitzberg DL, Caiaffa WT, Correia MI. Hospital malnutrition: the Brazilian national survey (IBRANUTRI): a study of 4000 patients. Nutrition. 2001:17:573-80.

Recebido em 20/10/2005. Aprovado em 26/1/2006. 